Abstract. We report a new approach for laparoscopic anatomical left lobectomy. Although laparoscopic limited resection of the liver has been reported, major liver surgery with a laparoscopic approach remains uncommon. Obstacles to routine laparoscopic surgery on the liver are mainly related to difficulty in retraction with current instrumentation, difficulty in assessing safe margins of resection without the use of tactile sense, and the difficulty of safe parenchymal dissection laparoscopically. We introduce a hand-assisted method that can help in resolving the difficulties and pitfalls associated with laparoscopic liver resection, and in making this surgery safer. The hand is the best atraumatic liver retractor in laparoscopic resection and facilitates the use of laparoscopic ultrasonography. Stable hemostasis can be achieved by proper manual application of vascular clips in case of vascular injury. The hemihepatic inflow control technique used in the present case was the en masse occlusion of Glisson's sheath of the left hemipedicle at the bifurcation. This technique was used exactly the same as in open surgery. Major vessels such as the left hemipedicle and left hepatic vein were dissected by endovascular cutter. The patient had an uneventful, quick postoperative recovery. This technique allows a minimally invasive anatomical major surgery for liver tumors.

Key words: Laparoscopic surgery — Left lobectomy of the liver - Hemihepatic vascular control technique - Hand-assisted method

Correspondence to: T. Kurokawa

\section{Pseudomonas aeruginosa liver abscesses after diagnostic endoscopic retrograde cholangiography in two patients with sphincter of Oddi dysfunction type 2}

P. Katsinelos, ${ }^{1}$ S. Dimiropoulos, ${ }^{1}$ D. Katsiba, ${ }^{2}$ M. Arvaniti, ${ }^{2}$

P. Tsolkas, ${ }^{1}$ I. Galanis, ${ }^{3}$ B. Papaziogas, ${ }^{3}$

V. Limenopoulos, ${ }^{1}$ S. Baltajiannis, ${ }^{1}$ I. Vasilladis ${ }^{1}$

${ }^{1}$ Department of Endoscopy and Motility Unit, Central Hospital, Ethnikis Aminis 41, TT 54635 Thessaloniki, Greece

${ }^{2}$ Department of Radiology, Central Hospital, Thessaloniki, Greece

${ }^{3}$ Second Department of Surgery, Aristotelion University, Thessaloniki, Greece

Received: 14 February 2002/Accepted in final form: 11 March 2002/ Online publication: 27 June 2002

DOI: $10.1007 / \mathrm{s} 00464-002-4210-9$

Abstract. Patients with sphincter of Oddi dysfunction have a significantly increased rate of pancreatitis after manometry or sphincterotomy, but septic complications after diagnostic endoscopic retrograde cholangiopancreatography (ERCP) in patients with sphincter of Oddi dysfunction type 2 have not been reported. We describe two patients with sphincter of Oddi dysfunction type 2 in whom Pseudomonas aeruginosa serotype 10 septicemia and multiple small hepatic abscesses developted, all within $48 \mathrm{~h}$ after they underwent diagnostic ERCP.

The sepsis and hepatic abscesses resolved after successful intravenous antibiotic administration. Despite scrupulous examination of the duodenoscope washing machine and the bottle of water, the bacteria responsible for the sepsis could not be isolated. It is possible that despite disinfection, a nondetectable colony of $P$. aeruginosa remained in a part of duodenoscope and proliferated to reach a potentially hazardous level the following day.
This report highlights the importance administering antibiotic prophylaxis to patients with sphincter Oddi dysfunction type 2 who undergo ERCP, despite the functional nature of the disease.

Key words: Pseudomonas aeruginosa - Hepatic abscesses - Sphincter of Oddi dysfunction - Endoscopic retrograde cholangiopancreatography (ERCP)

Correspondence to: P. Katsinelos

\section{Laparoscopic resection of patent omphalomesenteric duct in an adult}

\section{T. Z. Nursal, S. Yildirim, A. Tarim, T. Noyan}

Department of General Surgery, Başkent University Adana Teaching and Medical Research Center, Başkent Üniversitesi Adana Hastancsi, Dadaloğlu Mah, 39. Sok No:6, 01250 Yürcğir, Adana, Turkey

Received: 14 February 2002/Accepted in final form: 4 March 2002/ Online publication: 27 June 2002

DOI: $10.1007 / \mathrm{s} 00464-002-4209-2$

Abstract. The omphalomesenteric duct is the embryonic structure connecting the yolk sac to the primitive gut that disappears at 8 to 9 weeks of gestation. Failure of this duct to involute results in various anomalies. Failure of complete closure at different stages results in various anomalies, which occur in approximately $2 \%$ of the population. However, complete patency of omphalomesenteric duct is rare, reportedly occurring in approximately $15 \%$ omphalomesenteric duct anomalies. Adult presentations are extremely rare. We report the case of a 44-year-old man with a patent omphalomesenteric duct (POMD). He was admitted with a foul-smelling discharge from the umbilicus that he had experienced for 5 years. The condition had been interpreted as an umbilical granuloma at other centers, and multiple unsuccessful sessions of cauterization with silver-nitrate bar had been performed. At laparoscopy through three ports, the POMD was resected with the aid of a laparoscopic linear stapler. To our knowledge, this is the first adult case of POMD resected laparoscopically. The pitfalls of this diagnosis in the adult and the technique of laparoscopic resection is discussed in light of the available literature.

Key words: Laparoscopy - Omphalomesenteric duct - Stapler

Correspondence to: T. Z. Nursal

\section{Laparoscopic resection of perforated Meckel's diverticulum in a patient with clinical symptoms of acute appendicitis}

J. Ruh, ${ }^{1}$ A. Paul, ${ }^{1}$ O. Dirsch, ${ }^{2}$ M. Kaun, ${ }^{1}$ C. E. Broelsch ${ }^{1}$

${ }_{1}^{1}$ Department of General and Transplantation Surgery, University of Essen, Hufelandstrasse 55, 45122 Essen, Germany

${ }^{2}$ Department of Pathology, University of Essen, Hufelandstrasse 55, 45122 Essen, Germany

Received: 4 February 2002/Accepted in final form: 4 March 2002/

Online publication: 27 June 2002

DOI: $10.1007 / \mathrm{s} 00464-002-4207-4$

Abstract. We report the laparoscopic resection of a perforated Meckel's diverticulum (MD) found in a 14-year-old boy who presented with abdominal pain and nausea. There was rebound tenderness in the right lower quadrant of the abdo- 